\title{
Sham TMS: Intracerebral Measurement of the Induced Electrical Field and the Induction of Motor-Evoked Potentials
}

\author{
Sarah H. Lisanby, David Gutman, Bruce Luber, Charles Schroeder, and \\ Harold A. Sackeim
}

Testing the therapeutic potential of transcranial magnetic stimulation (TMS) in controlled trials requires a valid sham condition. Sham TMS is typically administered by tilting the coil $45-90^{\circ}$ off the scalp, with one or two wings of the coil touching the scalp. Lack of cortical effects has not been verified.

We compared sham manipulations in their thresholds for eliciting motor-evoked potentials (MEPS) in human volunteers and in intracerebral measurements of voltage induced in the prefrontal cortex of a rhesus monkey.

Three types of sham (one-wing $45^{\circ}$ and $90^{\circ}$ and twowing $90^{\circ}$ tilt) induced much lower voltage in the brain than active TMS (67-73\% reductions). However, the two-wing $45^{\circ}$ sham induced values just $24 \%$ below active TMS. This sham was about half as potent in inducing MEPs over the motor cortex as active TMS.

Some sham TMS conditions produce substantial cortical stimulation, making it critical to carefully select the sham manipulation for clinical trials. Biol Psychiatry 2001;49: 460-463 (C) 2001 Society of Biological Psychiatry

Key Words: Transcranial magnetic stimulation (TMS), sham, motor threshold (MT), depression, magnetics, induced current

\section{Introduction}

$\mathrm{S}$ everal controlled trials have found that transcranial magnetic stimulation (TMS) exerts antidepressant or antimanic effects (George et al 1997; Grisaru et al 1998; Klein et al 1999; Lisanby and Sackeim 2000; PascualLeone et al 1996). However, effect sizes have varied considerably, and some studies found no difference be-

\footnotetext{
From the Department of Biological Psychiatry, New York State Psychiatric Institute (SHL, DG, BL, HAS), and the Departments of Psychiatry (SHL, DG, BL, HAS) and Radiology (HAS), College of Physicians and Surgeons, Columbia University, New York; the Department of Neuroscience, Nathan S. Kline Institute for Psychiatric Research, Orangeburg (CS); and the Department of Neuroscience, Albert Einstein College of Medicine, Bronx (CS), New York Address reprint requests to Sarah H. Lisanby, M.D., New York State Psychiatric Institute, Department of Biological Psychiatry, 1051 Riverside Drive, Unit 126, New York NY 10032.

Received August 8, 2000; revised November 1, 2000; accepted November 14, 2000.
}

tween active TMS and sham stimulation (Loo et al 1999). In the Loo et al (1999) study, the lack of a differential effect between the active and sham treatments was seen in the presence of a substantial therapeutic response in the sham condition. Variability in therapeutic effects across studies may reflect differences in the biological activity of the sham manipulations employed. Validating the sham condition is key to determining whether TMS has therapeutic properties.

A valid sham should simulate the ancillary aspects of TMS (acoustic artifact, scalp muscle stimulation, daily experimenter contact, expectations about efficacy and side effects) but not result in cortical stimulation. The most common sham conditions angle the coil $45^{\circ}$ or $90^{\circ}$ off the head so that the magnetic field stimulates scalp muscles and produces an acoustic artifact, but presumably does not induce current in the cortex (Figure 1). In vivo validation of sham is critical. Indeed, Loo et al (2000) reported that a $45^{\circ}$ sham was about half as potent as standard TMS in stimulating the motor cortex.

In this study, electrical voltage induced in the brain with active and four types of sham TMS was measured intracerebrally in a rhesus monkey, representing the first in vivo measurements of TMS-induced intracerebral electric fields. We also titrated the threshold for excitation of the motor cortex in normal volunteers (motor threshold [MT]) as a noninvasive index of the cortical activity of various "inactive" sham conditions.

\section{Methods and Materials}

\section{Intracerebral Measurement of TMS-Induced Voltage}

This study was approved by our Institutional Animal Care and Use Committee. A nickel-chromium multicontact electrode (10 0.5 -mm contacts, $6 \mathrm{~mm}$ apart) was stereotaxically implanted in an anesthetized male Macaca mulatta. A remote occipital skull entry site was used to minimize the effect of the skull defect on TMS-induced current pathways in the prefrontal cortex. A postimplantation three-dimensional magnetic resonance image 


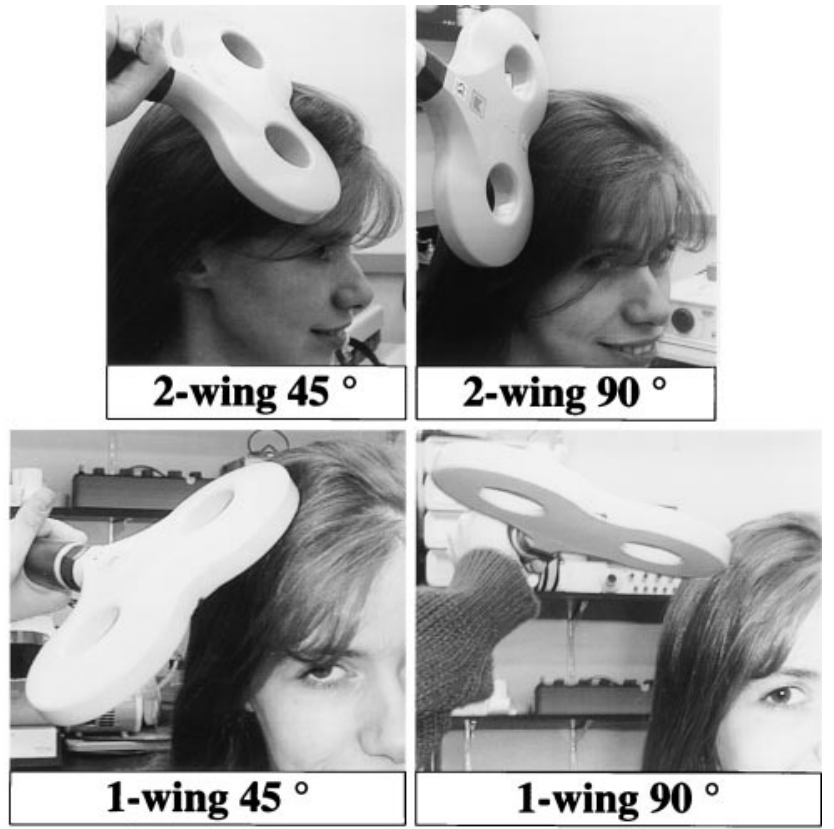

Figure 1. Coil positioning for several commonly used sham techniques. Active transcranial magnetic stimulation (TMS) is performed with the figure-eight coil tangential to the scalp, with the intersection of the figure eight in direct contact with the scalp. All of these sham manipulations consist of angling the coil slightly off the head such that the superficial scalp muscles are activated to simulate the sensation and acoustic artifact of repetitive TMS. This may be accomplished by tilting the coil such that two wings of the figure-eight coil touch the scalp (two-wing sham) or such that only one wing of the figure eight touches (one-wing sham). The degree of angulation from the plane tangential to the scalp is typically $45^{\circ}$ or $90^{\circ}$.

(GE [Milwaukee] Signa 1.5 T, contiguous 1.5-mm slices) was obtained to verify the electrode position in the prefrontal cortex relative to fiducial markers representing TMS coil position (Figure 1).

Potential differences $(\mu \mathrm{V})$ between each contact and an internal reference located in the occipital cortex were differentially amplified (Tektronix [Beaverton, OR] AM-501) and digitized at $250 \mathrm{kHz} / \mathrm{channel}$ to capture the dampened cosine waveform of the TMS-induced electrical pulse. Transcranial magnetic stimulation waveforms recorded intracerebrally matched those recorded in saline models and reversed polarity when the direction of induced current was reversed. Measured voltage across the contact sites was plotted to describe the voltage gradient induced by the magnetic pulse (McCreery et al 1990).

Active TMS and four types of sham (one or two wings of the figure-eight in contact with the scalp; $45^{\circ}$ or $90^{\circ}$ tilt from tangential) were applied with a figure-eight coil $(5 \mathrm{~cm}$ diameter) to the left prefrontal cortex, $3 \mathrm{~cm}$ anterior to the optimal site for stimulating the right first dorsal interosseous (FDI). Transcranial magnetic stimulation was administered with the Magstim (Whitland, United Kingdom) Super Rapid (1 Hz, 5-sec train, 70\% maximal output) with the subject under sedation (ketamine 15 $\mathrm{mg} / \mathrm{kg}$ and xylazine $1 \mathrm{mg} / \mathrm{kg}$ intramuscularly). Motor threshold under these conditions was $22 \%$ of maximal stimulator output.

\section{Motor Threshold with Active and Sham TMS}

Eight healthy normal adult volunteers (four female, mean age $29.6 \pm 1.6$ years, range $28-32$ ) were paid $\$ 10 /$ hour for participation. Subjects were excluded on the basis of age $>50$, history of DSM-IV axis I diagnosis, neurologic illness or head trauma, or use of prescription medication in the past month. No subject had previously received TMS, and all were masked to condition order. This study was approved by the Institutional Review Board of the New York State Psychiatric Institute. After complete description of the study, written informed consent was obtained.

Motor threshold was determined for active TMS and four sham conditions, administered in a randomized order. Tilt angle was held constant with a rigid wedge. Single-pulse TMS was delivered with the Magstim 200 (double 7-cm figure-eight coil). The optimal position for the FDI was defined as the scalp position eliciting the largest motor-evoked potential (MEP) and was marked on a swim cap. The coil handle was rotated $45^{\circ}$ from the parasagittal line such that the induced current would be in the optimal direction for stimulating the motor strip. Motor threshold was determined with voluntary contraction (10\% of maximal effort on a force transducer) to lower threshold and to serve as a more sensitive indicator of cortical stimulation. With a descending method of limits procedure with $2 \%$ steps, MT was defined as the minimal intensity that elicited an MEP of at least $50 \mu \mathrm{V}$ peak-to-peak amplitude averaged from five trials. At least $10 \mathrm{sec}$ were allowed between successive stimulations. Repeated-measures analysis of variance on MT values was used to test for main effects of coil angle and orientation. Post hoc paired $t$ tests were used to contrast the coil manipulation conditions.

\section{Results}

\section{Intracerebral Measurement of TMS-Induced Voltage}

Conforming to predictions (Cerri et al 1995), there was a rapid fall-off in the amplitude of the induced voltage with increasing distance from the coil. Amplitude was linearly related to TMS intensity $\left(r^{2}=.9, p<.0001\right)$.

Induced voltage differed as a function of TMS condition (Figure 2). Individual pulses within each 5-sec train showed extremely little amplitude variation (coefficient of variation $=2.4 \%$ ). All sham conditions induced substantially lower voltage than active TMS, and there was no overlap between the conditions. Peak integrated voltages of the TMS-induced dampened cosine waveform in the prefrontal cortex were $171.9 \pm 0.7 \mathrm{mV}$ for active TMS, $129.6 \pm 0.5 \mathrm{mV}$ for two-wing $45^{\circ}$ sham, $56.9 \pm 0.7 \mathrm{mV}$ for one-wing $45^{\circ}$ sham, $48.4 \pm 0.3 \mathrm{mV}$ for two-wing $90^{\circ}$ sham, and $50.0 \pm 0.2 \mathrm{mV}$ for one-wing $90^{\circ}$ sham. Although three of the sham conditions reduced the in- 


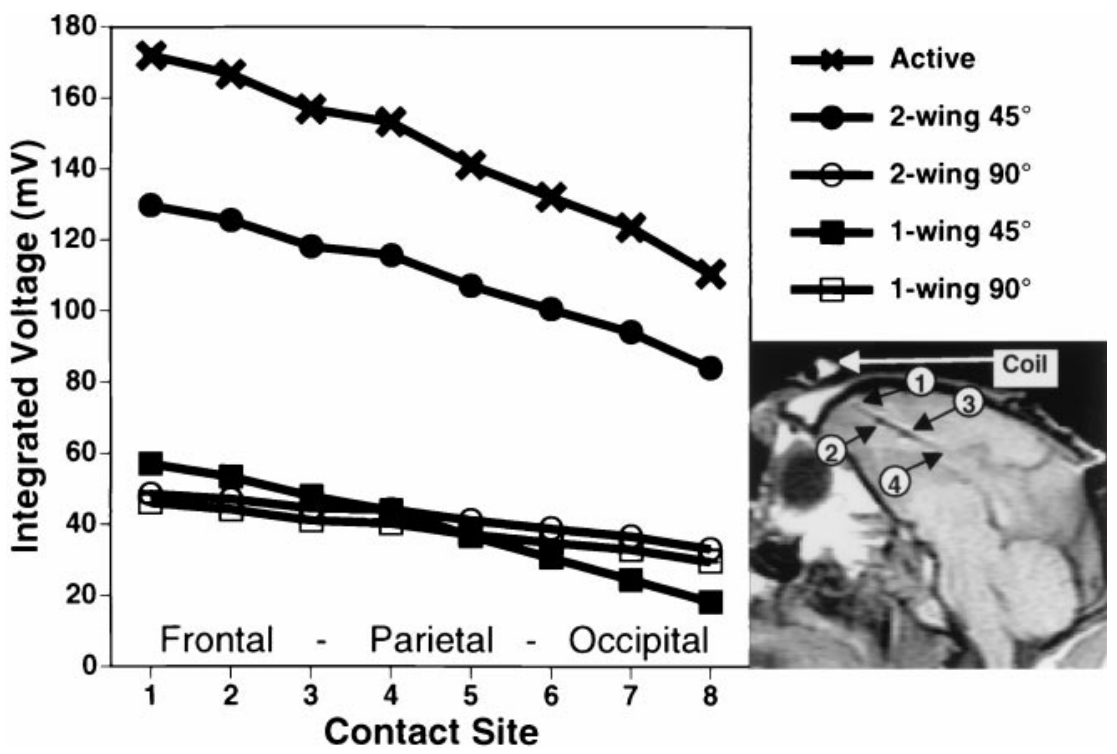

Figure 2. (Right) Intracerebral electrode contact sites marked on sagittal view, relative to fiducial marker of coil site. (Left) Intracerebral recordings of voltage induced in the brain in vivo by active and four types of sham transcranial magnetic stimulation.

duced field by $67-73 \%$, the two-wing $45^{\circ}$ orientation induced values that were only $24 \%$ below active TMS.

\section{Motor Threshold with Active and Sham TMS}

Mean MTs were $32 \% \pm 5 \%$ for active TMS, $66 \% \pm 12 \%$ for the two-wing $45^{\circ}$ sham, and $85 \% \pm 19 \%$ for the one-wing $45^{\circ}$ sham. Neither $90^{\circ}$ sham condition elicited MEPs, even at $100 \%$ maximal output. A repeated-measures analysis of variance on MT values demonstrated significant effects of coil angle $[F(1,7)=35.3, p<$ .0006], one wing versus two wings $[F(1,7)=6.3, p<$ $.04]$, and a wing by coil angle interaction $[F(1,7)=6.3$, $p<.04]$. The MT for the $45^{\circ}$ two-wing condition was approximately twice that for the active condition $[t(7)=$ $8.19, p<.0001]$, and the MT for the $45^{\circ}$ one-wing condition was 2.7 times active MT $[t(7)=7.47, p<$ $.0001]$.

\section{Discussion}

The $45^{\circ}$ sham conditions elicited substantial cortical stimulation at levels corresponding to $48-76 \%$ of active TMS, as evidenced by both MEPs in human subjects and measurements of intracerebral voltage in a nonhuman primate. In agreement with Loo et al (2000), the effects of the $45^{\circ}$ sham were most marked for the two-wing condition. Although Loo et al (2000) tested only the $45^{\circ}$ sham, we demonstrate that the $90^{\circ}$ sham (both one and two wing) conditions are devoid of biological effects as measurable in this study, and present the first in vivo measurements comparing active and sham repetitive TMS (rTMS)induced intracerebral voltage.

These findings raise the possibility that some sham conditions may be biologically active. This could artificially increase therapeutic response to sham treatment, and may in part explain some of the variability in effect sizes across studies. The relevance of stimulation below the MT is an important issue. It is conceivable that repetitive stimulation of the prefrontal cortex at levels below the threshold for motor cortex excitation to a single pulse may have a clinical effect. In fact, several studies have found antidepressant efficacy with repetitive stimulation at $80 \%$ of MT (George et al 1997). This observation also implies a possible role for summation from one pulse to the next, and these cumulative effects may be frequency dependent, as illustrated in the cases of rTMS-induced seizures (Wassermann et al 1996). Further evidence that stimulation below the MT may have observable effects is found in the phenomena of paired pulse inhibition and facilitation. A subthreshold conditioning pulse can either enhance or inhibit the cortical response to a subsequent suprathreshold pulse, contingent upon the interstimulus interval (Ziemann 1999).

A limitation of this study was the lack of ratings of the subjective effects of the various sham conditions. Instead, we focused on contrasting these conditions in induced voltage and biological activity. Another limitation is that MT assesses depolarization in the motor cortex, whereas most studies in depression and mania involve the prefrontal cortex. However, our in vivo measurements of induced voltage sampled the prefrontal cortex and substantiate the MT findings.

Given these findings, we recommend tilting the coil $90^{\circ}$ from tangential rather than the $45^{\circ}$ sham to minimize cortical effects. Neither the one-wing nor the two-wing $90^{\circ}$ sham elicited MEPs, even at maximal stimulator 
output. It should be acknowledged, however, that the $90^{\circ}$ sham may feel less intense subjectively, but this should be less of a problem for between-subject studies than withinsubject crossover designs. The correspondence seen between the in vitro recordings in monkeys and MT results in humans suggests that evaluating the MT with the sham condition selected may represent a readily available, though approximate, means of testing its biological activity. Such testing may be advisable because the output characteristics of coils differ across specific coil designs and manufacturers.

Supported in part by National Institute of Mental Health Grant No. MH01577 and a Young Investigator Award from the National Alliance for Research on Schizophrenia and Depression.

\section{References}

Cerri G, De Leo F, Moglie F, Schiavoni A (1995): An accurate 3-D model for magnetic stimulation of the brain cortex. J Med Eng Technol 19:7-16.

George MS, Wassermann EM, Kimbrell TA, Little JT, Williams WE, Danielson AL, et al (1997): Mood improvement following daily left prefrontal repetitive transcranial magnetic stimulation in patients with depression: A placebo-controlled crossover trial. Am J Psychiatry 154:1752-1756.

Grisaru N, Chudakov B, Yaroslavsky Y, Belmaker RH (1998): TMS in mania: A controlled study. Am J Psychol 155:16081610.
Klein E, Kreinin I, Chistyokov A, Koren D, Mecz L, Marmur S, et al (1999): Therapeutic efficacy of right prefrontal slow repetitive transcranial magnetic stimulation in major depression: A double-blind controlled study. Arch Gen Psychiatry 56:315-320.

Lisanby SH, Sackeim HA (2000): TMS in major depression. In: George MS, Belmaker RH, editors. Transcranial Magnetic Stimulation (TMS): Applications in Neuropsychiatry. Washington, DC: American Psychiatric Press, 185-200.

Loo C, Mitchell P, Sachdev P, McDarmont B, Parker G, Gandevia S (1999): Double-blind controlled investigation of transcranial magnetic stimulation for the treatment of resistant major depression. Am J Psychiatry 156:946-948.

Loo CK, Taylor JL, Gandevia SC, McDarmont BN, Mitchell PB, Sachdev PS (2000): Transcranial magnetic stimulation (TMS) in controlled treatment studies: Are some "sham" forms active? Biol Psychiatry 47:325-331.

McCreery DB, Agnew WF, Yuen TGH, Bullara L (1990): Charge density and charge per phase as cofactors in neural injury induced by electrical stimulation. IEEE Trans Biomed Eng 37:996-1001.

Pascual-Leone A, Rubio B, Pallardo F, Catala MD (1996): Rapid-rate transcranial magnetic stimulation of left dorsolateral prefrontal cortex in drug-resistant depression. Lancet 348:233-237.

Wassermann EM, Cohen LG, Flitman SS, Chen R, Hallett M (1996): Seizures in healthy people with repeated "safe" trains of transcranial magnetic stimuli. Lancet 347:825-826.

Ziemann U (1999): Intracortical inhibition and facilitation in the conventional paired TMS paradigm. Electroencephalogr Clin Neurophysiol Suppl 51:127-136. 